\title{
Effect of Poly(amic diethyl ester) Precursor on Residual Stress Behavior of Aromatic Polyimides
}

\author{
Hyunsoo Chung, Yung-Il JoE, and Haksoo $\mathrm{HAN}^{\dagger}$ \\ Department of Chemical Engineering, Yonsei University, \\ 134 Shinchon-Dong, Sedaemun-Gu, Seoul 120-749. Korea
}

(Received November 6, 1998)

\begin{abstract}
The influence of poly(amic diethyl ester) (PAE) and poly(amic acid) (PAA) precursors on the residual stress behavior of polyimide thin films was investigated. Rodlike poly( $p$-phenylene pymellitimide) (PMDA-PDA), pseudo-rodlike poly( $p$-phenylene biphenyltetracarboximide) (BPDA-PDA), semiflexible poly(4,4-oxydiphenylene pyromellitimide), and poly(4,4-oxydiphenylene biphenyltetracarboximide) (BPDA-ODA) were used. For polyimides prepared from PAE precursor, residual stress of fully cured films at $400 \mathrm{C}$ were $13.4 \mathrm{MPa}$ for the PMDA-PDA, 19.5 MPa for BPDA-PDA, 30.3 MPa for PMDA-ODA, and 39.5 MPa for BPDA-ODA. For polyimides prepared from PAA precursor, residual stress after fully cured at $400^{\circ} \mathrm{C}$ was $-5.1 \mathrm{MPa}$ for the PMDA-PDA, 7.0 MPa for BPDA-PDA, 29.4 MPa for PMDA-ODA, and 40.1 MPa for BPDA-ODA. The effect of different size of precursor on the residual stress behavior was significantly high for rodlike polyimides having high chain rigidity and a high intermolecular packing order, but relatively low for the semi-flexible polyimides with relatively low chain rigidity. The degree of molecular order for the rigid structure polyimides, PMDA-PDA and BPDA-PDA, was hindered by the bulky ethyl ester group evaporated during thermal imidization. However, the effect of the bulky ethyl ester group was not significant for the flexible structure polyimides, PMDA-ODA and BPDA-ODA. Residual stress was in decreasing order BPDA-ODA < PMDA-ODA < BPDA-PDA < PMDA-PDA polyimide regardless of the precursor origins. These results indicate that the residual stress behavior in the polyimides depending on precursor origin is significantly related to the morphological structure as well as the chain mobility of the polyimide during thermal imidization.
\end{abstract} KEY WORDS Residual Stress/Precursor Origin/Morphology/Chain Mobility

Polyimides are used as interlayer dielectrics, passivation layers and alpha particle barriers because of high thermal stability, relatively low dielectric constant, good mechanical properties, and high chemical resistance. ${ }^{1-3}$ They are insoluble in most solvents so that they are processed in soluble precursors and imidized thermally or chemically. One polyimide precursor is PAA which is easily synthesized in an aprotic solvent from the polycondensation of dianhydride and diamine monomers. However, another polyimide precursor is PAE which has increased hydrolytic stability in solution, improved solubility, higher imidization temperature regime that offers a wider processing window for good adhesion. ${ }^{4,5}$ Thus, in spite of inconvenience in the synthesis, PAE as a polyimide precursor is more adequate to fabrication in the microelectronic industry.

The thermal imidization behavior of PAE differs from that of PAA. ${ }^{6,7}$ The different imidization kinetics between precursors of a polyimide may lead to different morphological structures and properties in the resultant polyimide even though both the precursors provide the chemically identical polyimide. For application of poly(amic dialkyl ester)s (PAEs) in the fabrication of devices, residual stress is one of the most important properties to be investigated because residual stress causes delamination at the metal/polyimide interface or a crack formation in the metal or in the polyimide film associated with reliability problems. ${ }^{8,9}$ The properties of the polyimide derived from a PAE may be different from those of the respective polyimide, even though they have identical chemical backbones by the conventional thermal baking. Outgassing of the ethyl groups and the package components depends strongly upon the imi- dization conditions as well as boiling points and degradation temperatures. Even though ethyl groups are fully outgassed, they may influence the morphological structure of the precursor in the condensed state as well as imidization kinetics, consequently leading to different morphologies and properties in the resultant polyimide. The residual stress behavior of polyimide-PAA derived films has been extensively studied using bending beam methods and wafer methods. ${ }^{10-14}$ Residual stress in polyimide films occurs apparently by the thermal mismatch between $\mathrm{Si}$ wafer and polyimide thin films. Ree et al. ${ }^{13,14}$ found that the residual stress in polyimide film was influenced by chain rigidity, in-plane orientation, and thickness. However, residual stress for some of polyimide prepared from PAE precursor has been rarely investigated except Ree et al. Residual stress in PAE-derived film is not yet fully understood with morphological structure. Therefore, in the present study, PAEs of four different polyimides with various chain rigidities were synthesized. Residual stress behavior of the polyimide films adhering to silicon substrates, due to thermal mismatch, was in situ measured using a thin film stress analyzer (TFSA) during thermal imidization. Wide-angle X-ray diffraction and dynamic mechanical thermal analysis were used for morphological structures and chain mobility of polyimide film during thermal imidization.

\section{EXPERIMENTAL}

\section{Material and Sample Preparation}

Poly(amic acid)s (PAAs) were synthesized in dry NMP from the respective dianhydrides and diamines: poly-

${ }^{\dagger}$ To whom correspondence should be addressed (Tel: +82-2-361-2764, Fax: +82-2-312-6401, e-mail: hshan (a,yonsei.ac.kr). 


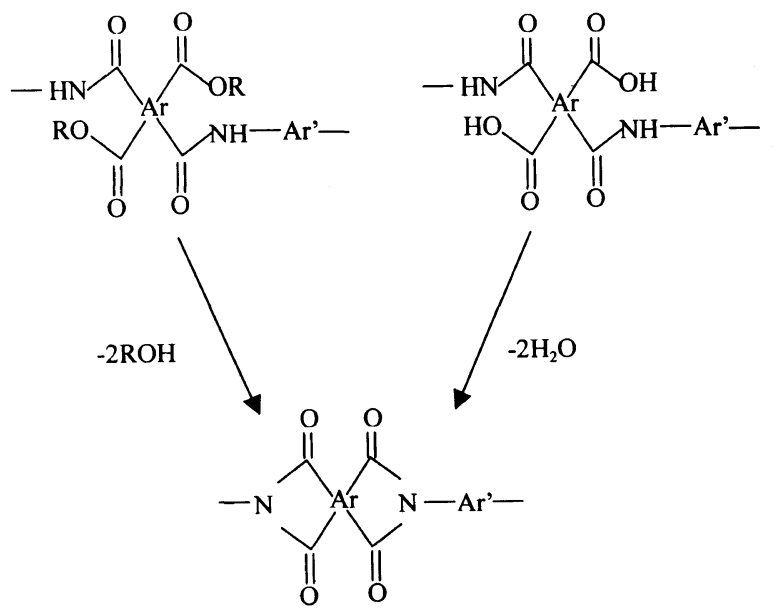

Figure 1. Polyimide prepared from their poly(amic diethyl ester) (PAE) and poly(amic acid) (PAA) precursors by thermal imidization: here, $\mathrm{R}$ is ethyl.

( $p$-phenylene pyromellitamic acid) (PMDA-PDA PAA), poly $(p$-phenylene biphenyltetracarboxamic acid) (BPDA-PDA PAA), poly(4,4'-oxydiphenylene pyromellitamic acid) (PMDA-ODA PAA), and poly $\left(4,4^{\prime}\right.$ oxydiphenylene biphenyltetracarboxamic acid) (BPDAODA PAA). The corresponding poly( $p$-phenylene pyromellitamic diethyl ester) (PMDA-PDA PAE) was synthesized by low temperature polymerization of $p$ phenylene and pyromellitic diethyl ester diacyl in dry $N$-methyl-2-pyrrolidone (NMP). ${ }^{4,15,16}$ The precursor polymer solution was precipitated in deionized water, filtered, and washed with methanol to remove the $\mathrm{HCl}$ by-products. The polymer solution was dried for 2 days at $50 \mathrm{C}$ in a vacuum oven. The other PAEs were prepared with the same method: poly( $p$-phenylene biphenyltetra carboxamic diethyl ester) (BPDA-PDA PAE), poly $\left(4,4^{\prime}\right.$ oxydiphenylene pyromellitamic diethyl ester) (PMDAODA PAE), and poly (4,4'-oxydiphenylene biphenyltetracarboxamic diethyl ester) (BPDA-ODA PAE). All monomers and chemicals were supported from Aldrich Co.

\section{Measurement}

The one-side polished $\mathrm{Si}$ (100) wafers as substrates used in this study were $76.2 \mathrm{~mm}$ in diameter. For the polyimide thin films, precursor solutions with concentration of $12-15 \mathrm{wt} \%$ were spin-coated on silicon substrates, following soft baking on hot plate at $80^{\circ} \mathrm{C}$ for $1 \mathrm{~h}$. During thermal imidization from $25-400^{\circ} \mathrm{C}$, the residual stress behavior of polyimide films was monitored using a TFSA. The ramping rate was $2.0^{\circ} \mathrm{C} \mathrm{min}^{-1}$ and cooling rate was $1.0^{\circ} \mathrm{C} \mathrm{min}^{-1}$. The thickness of polyimide films was controlled at $9-11 \mu \mathrm{m}$ to exclude thickness effect of the sample.

To determine the residual stress of the polyimide thin films from the measured radii of curvature, eq 1 was used. $^{17}$

$$
\sigma=\frac{E_{\mathrm{s}}}{6\left(1-v_{\mathrm{s}}\right)} \frac{t_{\mathrm{s}}^{2}}{t_{\mathrm{f}}}\left(\frac{1}{R_{2}}-\frac{1}{R_{2}}\right)
$$

$\sigma$ is residual stress in the polyimide film. The subscripts, f and $\mathrm{s}$ denote the polyimide film and substrate. $E, v$,

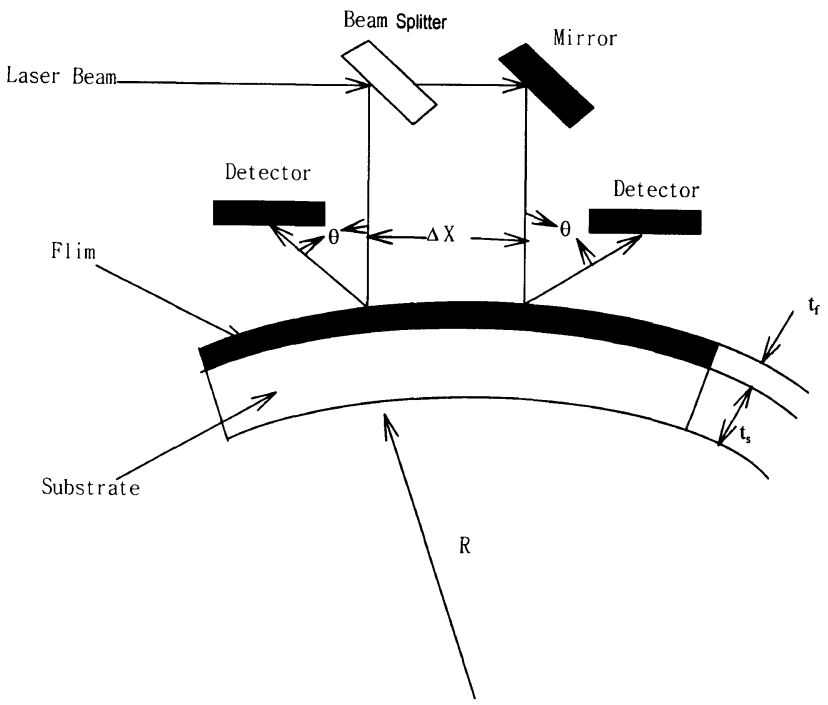

Figure 2. Thin film stress apparatus (TFSA).

and $t$ are Young's modulus, Poisson's ratio, and the thickness of the substrate, respectively. $R_{1}$ and $R_{2}$ are wafer curvatures measured before and after film deposition. For Si $(100)$ wafer, $E_{\mathrm{s}} /\left(1-v_{\mathrm{s}}\right)$ is $180500 \mathrm{MPa}{ }^{18}$ Before use, initial curvature and thickness of wafers were measured using TFSA and the thickness gauge (SM1201, Teclock Co., Japan).

For morphological and other characteristic properties of the polyimide, fully cured polyimide films were removed from the silicon substrate after soaking in distilled water for $24 \mathrm{~h}$. They were washed with distilled water several times and dried. Wide angle X-ray diffraction (WAXD) measurement was made for the morphological structure of polyimide thin films. It was conducted by the $\theta / 2 \theta$ method over $3.5-60^{\circ}$ using a Rigaku diffractometer with $\mathrm{Cu}-K_{\alpha}(\lambda=1.54 \AA)$ radiation source. Step and count data were taken at $0.02^{\circ}$ intervals a scan speed of $0.4^{\circ} \mathrm{min}^{-1}$. Measured WAXD was corrected to the background run and normalized for the polyimide thin film prepared form PAE and the corresponding PAA precursor by matching the integrated intensity over the range $58-60^{\circ}(2 \theta) .{ }^{19-21}$

For the glass transition properties of polyimide thin films, dynamic mechanical thermal analyzer (DMTA) (Polymer Lab., MKIII) was used. The heating rate and frequency were $5.0^{\circ} \mathrm{Cmin}^{-1}$ and $1 \mathrm{~Hz}$, respectively.

\section{RESULTS AND DISCUSSION}

The residual stress of four backbone structure polyimides was measured as shown in Table I. For rodlike PMDA-PDA, the residual stress of fully cured polyimide thin film prepared from the PAA precursor was $-5.1 \mathrm{MPa}$ at $25^{\circ} \mathrm{C}$. Surprisingly, the residual stress of PMDA-PDA polyimide thin film prepared from the PAE precursor was $13.4 \mathrm{MPa}$ after fully cured at $400^{\circ} \mathrm{C}$ for $1 \mathrm{~h}$. For the pseudo-rodlike BPDA-PDA polyimide, the effect of diethyl ester precursor on the residual stress was similarly observed. However, the residual stress behavior for semiflexible structure PMDA-ODA and BPDA-ODA depending on precursor origin is relatively insensitive compared to that of rigid structure PMDA-PDA and 
Table I. Residual stress of polyimides prepared from poly(amic diethyl ester) (PAE) and poly(amic acid) (PAA) precursors

\begin{tabular}{|c|c|c|c|c|}
\hline \multirow{2}{*}{ Polyimide } & \multirow{2}{*}{$\begin{array}{l}\text { Precursor } \\
\text { origin }\end{array}$} & \multirow{2}{*}{$\begin{array}{c}\text { Film thickness } \\
\qquad \mu \mathrm{m}\end{array}$} & \multirow{2}{*}{$\frac{\text { Stress at } 400^{\circ} \mathrm{C}}{\mathrm{MPa}}$} & \multirow{2}{*}{$\begin{array}{l}\text { Residual stress at } 25^{\circ} \mathrm{C} \\
\text { after fully cure } / \mathrm{MPa}\end{array}$} \\
\hline & & & & \\
\hline PMDA-PDA & PAE & 10.5 & 3.5 & 13.4 \\
\hline \multirow{2}{*}{ BPDA-PDA } & PAA & 9.8 & 4.3 & 7.0 \\
\hline & PAE & 10.1 & 2.7 & 19.5 \\
\hline \multirow{2}{*}{ PMDA-ODA } & PAA & 10.5 & 0 & 29.4 \\
\hline & PAE & 12.0 & 0 & 30.3 \\
\hline \multirow{2}{*}{ BPDA-ODA } & PAA & 10.2 & 0 & 40.1 \\
\hline & PAE & 10.3 & 0 & 39.5 \\
\hline
\end{tabular}

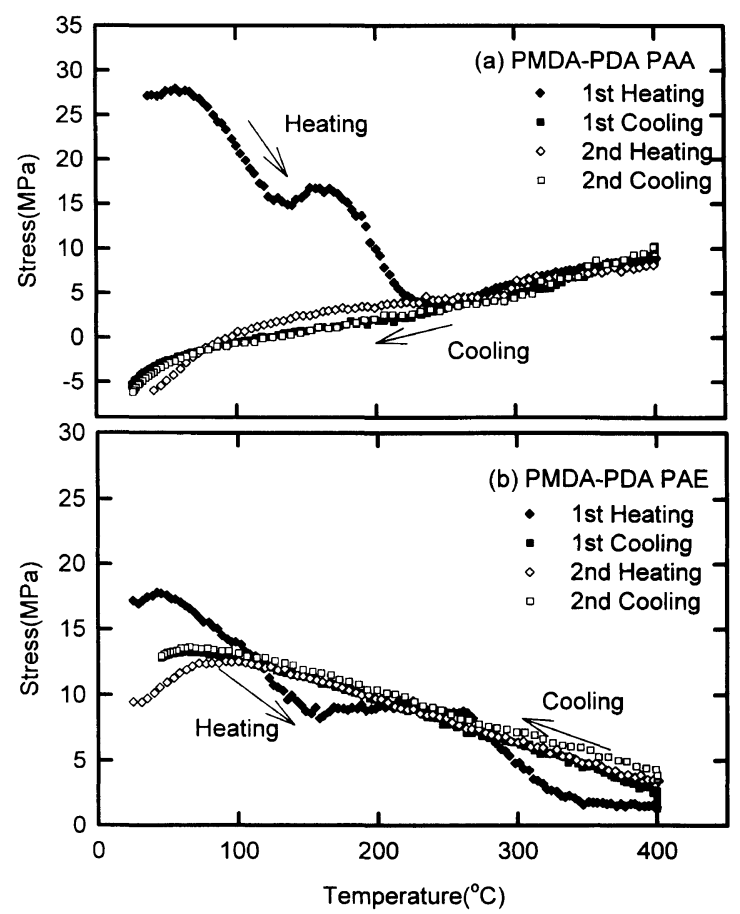

Figure 3. Residual stress behavior of PMDA-PDA thin films prepared from poly(amic diethyl ester) (PAE) and poly(amic acid) (PAA).

BPDA-PDA as shown in Table I. That is, the effect of precursor origin on residual stress behavior for fully cured polyimide film was negligible for flexible PMDAODA and BPDA-ODA.

As shown in Figure 3, stress behavior for the rodlike PMDA-PDA derived from PAE precursor is quite different from that of the polyimide prepared from PMDA-PDA PAA precursor. The stress of prebaked precursor films at $80^{\circ} \mathrm{C}$ initially decreased with increasing temperature until imidization of polyimide. The stress of polyimide thin film relatively increased on further elevating temperature above $130^{\circ} \mathrm{C}$, reflecting evaporation of residual solvent and the imidization. Resulting stress at $400^{\circ} \mathrm{C}$ of polyimides prepared from the PMDA-PDA PAA and the PMDA-PDA PAE was $9.1 \mathrm{MPa}$ and $3.5 \mathrm{MPa}$, respectively. In cooling from $400^{\circ} \mathrm{C}$ to $25^{\circ} \mathrm{C}$, residual stress of the PMDA-PDA polyimide films prepared from PAA precursor decreas702

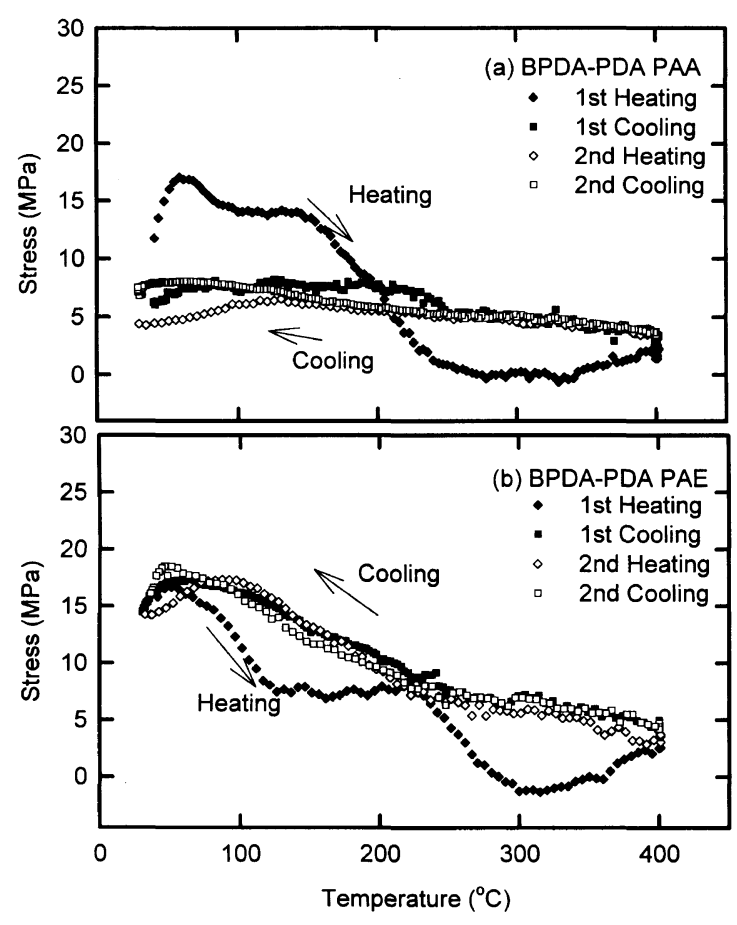

Figure 4. Residual stress of BPDA-PDA thin films prepared from poly(amic diethyl ester) (PAE) and poly(amic acid) (PAA).

ed with decreasing temperature but increased in the polyimide film prepared from PMDA-PDA PAE. The residual stress at $25^{\circ} \mathrm{C}$ of fully cured polyimides prepared from the PMDA-PDA PAA and the PMDA-PDA PAE was $-5.1 \mathrm{MPa}$ and $13.4 \mathrm{MPa}$, respectively. Specially, the stress of polyimide prepared from the PMDA-PDA PAE is in the tension mode over the range of $25-400^{\circ} \mathrm{C}$. The thermal expansion coefficient (TEC) is thus always greater than that of $\mathrm{Si}(100)\left(3 \mathrm{ppm} /{ }^{\circ} \mathrm{C}\right) .{ }^{22}$ Here, the negative sign appeared in the PMDA-PDA prepared from PAA indicates stress in compression mode. ${ }^{23}$

For pseudo-rodlike BPDA-PDA, after softbake at $80^{\circ} \mathrm{C}$, the stress at $25^{\circ} \mathrm{C}$ polyimides prepared from the BPDA-PDA PAA and the BPDA-PDA PAE was $15 \mathrm{MPa}$ and $14 \mathrm{MPa}$ as shown in Figure 4. At $400^{\circ} \mathrm{C}$ during curing, the stress of polyimides prepared from the BPDA-PDA PAA and BPDA-PDA PAE was $4.3 \mathrm{MPa}$ and $2.7 \mathrm{MPa}$, respectively. The stress of $\mathrm{BPDA}-\mathrm{PDA}$ polyimide thin films at $400^{\circ} \mathrm{C}$ was not relatively sensi- 


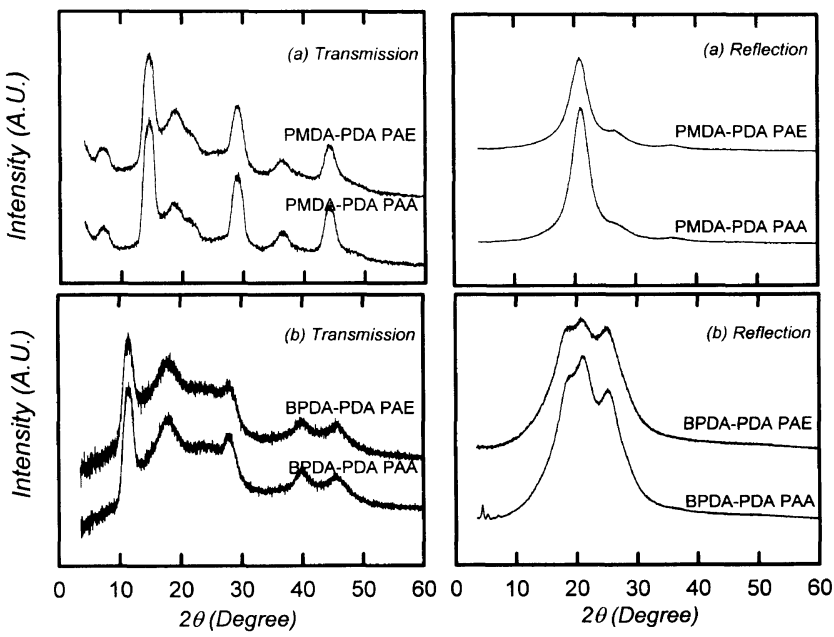

Figure 5. WAXD of polyimide films prepared from poly(amic diethyl ester) (PAE) and poly(amic acid) (PAA) precursors: (a) PMDA-PDA: (b) BPDA-PDA.

tive to the precursor origin compared to that of PMDAPDA. Thus, the BPDA-PDA polyimide chains at the $400 \mathrm{C}$ are relatively more mobile than rodlike PMDAPDA, resulting in a tension stress regardless of precursor origin. This may correlate with the glass transition behavior of polyimide chain. This molecular mobility is very much restricted in the PMDA-PDA polyimide, which does not exhibit glass transition over the considered temperature range,,$^{2,24,25}$ so that stress cannot be easily minimized during curing. In cooling, the BPDA-PDA polyimide showed significantly different stress behavior depending on the precursor origin. For the polyimide prepared from BPDA-PDA PAA precursor, the residual stress of the fully cured polyimide increased slowly with decreasing temperature and reached $7 \mathrm{MPa}$ at $25^{\circ} \mathrm{C}$. However, for the polyimide prepared from BPDA-PDA PAE, the stress of the resulting polyimide built up rapidly with decreasing temperature and reached $19.5 \mathrm{MPa}$ at $25^{\circ} \mathrm{C}$. This suggests that the evaporation of large molecular size of diethyl groups in BPDA-PDA PAE precursor severely affects the stress of the resulting BPDA-PDA polyimide. And residual stress behavior of the pseudo-rodlike BPDAPDA polyimide with rodlike PMDA-PDA polyimide is sensitive to the precursor origin on cooling.

PAE-derived polyimide films, PMDA-PDA and BPDA-PDA show a higher thermal stress variation than that of the PAA-derived ones. This indicates the PAE-derived films to have a higher biaxial modulus and/or coefficient of thermal expansion. It may indicate that the outgassing diethyl groups disturb the molecular chain order in the resulting polyimide film. This may show that the change of morphology (chain orientation and molecular order) in polyimide leads significant the thermal mismatch and the residual stress due to precursor origin.

For the rodlike structure PMDA-PDA and pseudo rodlike structure BPDA-PDA polyimides, the morphological change of polyimide prepared from amic acid and diethyl ester precursors was investigated using WAXD as shown in Figure 5. The transmission of PMDA-PDA polyimide apparently shows multiple diffraction peaks from the high chain ordering along the chain axis, but exhibits only on amorphous halo peak in the reflection in Figure 5(a). In comparison to the pseudo-rodlike structure BPDA-PDA polyimide, PMDA-PDA chains are highly oriented in the film plane and highly ordered due to the rodlike chain, but are irregularly packed together. The lack of regular intermolecular packing might be the result of the limited chain mobility due to higher chain rigidity of PMDA-PDA. This may be related to the thermal properties of polymer such as glass transition temperature. The PAA-derived PMDA-PDA polyimide film exhibited relatively sharper and stronger diffraction peaks than the PAE-derived film as shown in Figure 5(a). In the reflection peak, the amorphous halo is much sharper and stronger in intensity for the PAAderived polyimide film than for the PAE-derived film. Coherence length, as the key factor representing chain order along the chain axis was estimated are $130 \AA$ for the PAA-derived film and $120 \AA$ for the PAE-derived film from the peak maximum of $(00 l)$ peak. Larger molecule evaporation during imidization disrupt the ordered phase in the PMDA-PDA polyimide prepared from diethyl ester precursor.

As observed for PMDA-PDA film, multiple $(00 l)$ peaks appeared only in the transmission of pseudo-rodlike BPDA-PDA, indicating that highly ordered molecules along chain axis are preferentially aligned in the film plane in Figure 5(b). The $(00 l)$ peaks are $(004)$ peak at $11.10^{\circ},(0010)$ at $27.90^{\circ},(0014)$ at $39.76^{\circ}$, and $(0016)$ at $45.18^{\circ}$, respectively. The PAA-derived film revealed a high intermolecular packing order and higher overall crystallinity than the PAE-derived film in BPDAPDA. Estimated coherence lengths were $123 \AA$ for the PAA-derived polyimide film and $118 \AA$ for the PAEderived film from the peak maximum of $(00 l)$ peak. For rodlike PMDA-PDA and pseudo-rodlike BPDA-PDA, PAA precursors produced better chain rigidity and higher population of more ordered phase in the resultant polyimide film than the corresponding PAE precursors as described above. These morphological differences may be reflected directly on the residual stress, consequently leading to a higher residual stress in the PAE-derived polyimide films than the PAA-derived films.

The residual stress behavior of semiflexible structure PMDA-ODA and BPDA-ODA was quite different with those of rigid structure PMDA-PDA and BPDA-PDA. In Figure 6, for semiflexible PMDA-ODA polyimides, stress at $25^{\circ} \mathrm{C}$ polyimides prepared from the PMDAODA PAA and PMDA-ODA PAE was $12.2 \mathrm{MPa}$ and $10 \mathrm{MPa}$. The residual stress of polyimide films was nearly $0 \mathrm{MPa}$, at $400^{\circ} \mathrm{C}$, regardless of precursor origin. This means that at $400^{\circ} \mathrm{C}$, the degree of the molecular mobility in the polyimide film is relatively higher than rigid polyimides. The completely released stress at $400^{\circ} \mathrm{C}$ indicates the glass transition state of PMDA-ODA because the chain mobility is directly related to the $T_{\mathrm{g}}$. In cooling after cure, stress increased with decreasing temperature regardless of precursor type. That is, the PMDA-ODA polyimide did not show any strong dependency of the precursor origin on stress behavior in contrast to both rodlike PMDA-PDA and pseudorodlike BPDA-PDA polyimides. The residual stress at $25^{\circ} \mathrm{C}$ of fully cured polyimides prepared from the PMDA- 


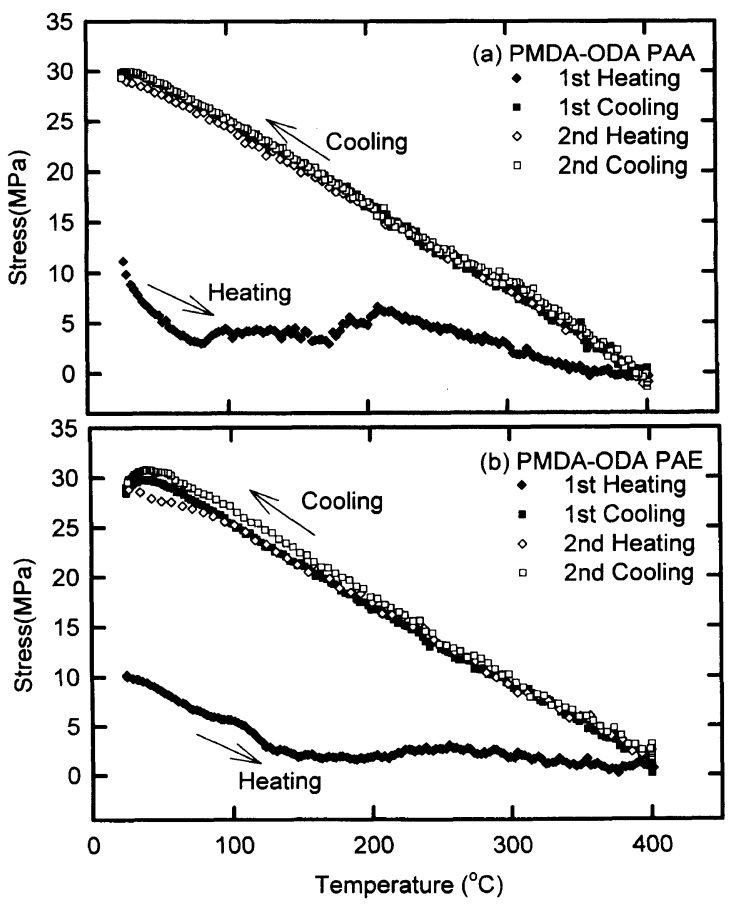

Figure 6. Residual stress of PMDA-ODA thin films prepared from poly(amic diethyl ester) (PAE) and poly(amic acid) (PAA).

ODA PAA and PMDA-ODA PAE was $29.4 \mathrm{MPa}$ and $30.3 \mathrm{MPa}$, respectively. Outgassing diethyl group during thermal imidization is thus less significant in flexible structure such as PMDA-ODA. This may result from the poor molecular orientation due to its hinged structure with ODA.

For BPDA-ODA PAA and BPDA-ODA PAE precursors, stress-temperature profiles are shown in Figure 7. After prebake at $80^{\circ} \mathrm{C}$, residual stress was $15 \mathrm{MPa}$ regardless of precursor origin. After curing, stress at $400^{\circ} \mathrm{C}$ was $0 \mathrm{MPa}$ for the polyimide films from the precursors. On cooling from $400^{\circ} \mathrm{C}$, stress in the polyimide films from both precursors remained at almost $0 \mathrm{MPa}$ until $290^{\circ} \mathrm{C}$, and thereafter increased rapidly by restricted chain mobility, indicating that $T_{\mathrm{g}}$ of the resulting BPDA-ODA polyimide films was about $290^{\circ} \mathrm{C}$. Residual stress at $25^{\circ} \mathrm{C}$ of fully cured polyimides prepared from the PMDA-PDA PAA and the PMDA-PDA PAE was $40.1 \mathrm{MPa}$ and $39.5 \mathrm{MPa}$, respectively. As observed on the semiflexible PMDA-ODA polyimide, the BPDAODA polyimide did not show significant dependency of the precursor on stress temperature.

This suggests that the effect of precursor origin on the morphological structure is relatively smaller in PMDAODA and BPDA-ODA than in PMDA-PDA and the BPDA-PDA film. Molecular chain orientation would be relatively low even in fully extended flexible polyimide because of the bend chain conformation due to the ether linkage of ODA diamine unit on the backbone linkage in PMDA-ODA and BPDA-ODA.

Morphological changes in these flexible PMDA-ODA and BPDA-ODA polyimides were investigated by WAXD. As shown in Figure 8(a), PMDA-ODA exhibited a sharp diffraction peak at the low angle region corresponding to the chain order along the chain axis, and a big amorphous halo in the transmission peak, but only the big amorphous halo peak in the reflection

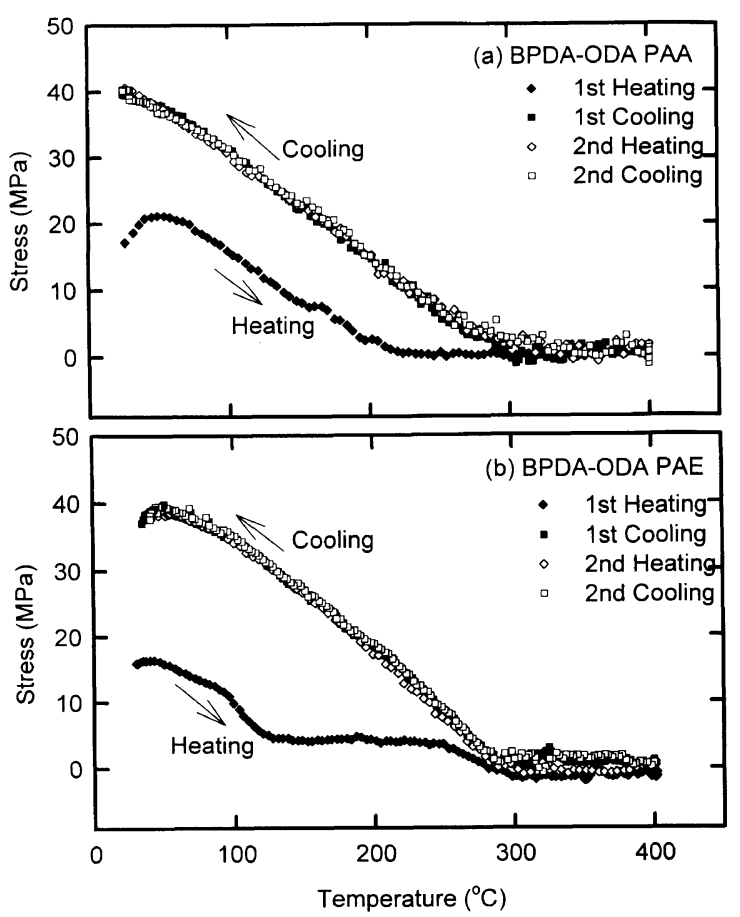

Figure 7. Residual stress behavior of BPDA-ODA thin films prepared from poly(amic diethyl ester) (PAE) and poly(amic acid) (PAA).
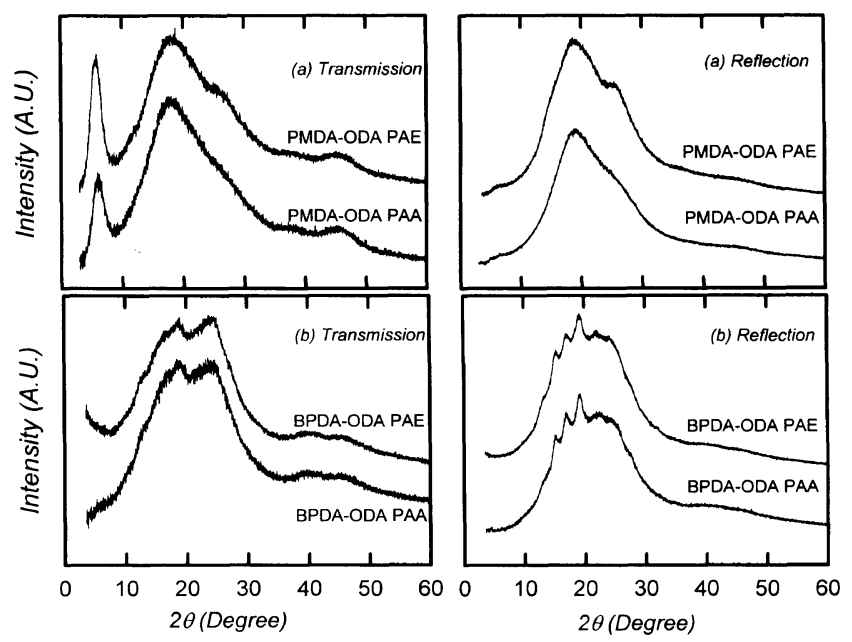

Figure 8. WAXD of polyimide films prepared from poly(amic diethyl ester) (PAE) and poly(amic acid) (PAA) precursors: (a) PMDA-ODA; (b) BPDA-ODA.

pattern. This means that PMDA-ODA has somewhat chain order along the chain axis, but is irregularly packed in the direction of film thickness. The PAE precursor produced a relatively better intermolecular order in the resultant polyimide film than the corresponding PAA precursor. The intensity of (002) diffraction peak in the transmission increased with PAEderived film, confirming increase in the molecular in plain orientation. However, the coherence length for the (002) peak, which represents the degree of molecular chain order along the chain axis, is $80-85 \AA$, nearly insensitive to precursor origin. Thus, similar stress in PAE-derived PMDA-ODA film might result mainly from the similar chain rigidity in PAA-derived film due to ODA diamine.

The effect of ethyl ester group was negligible in the 

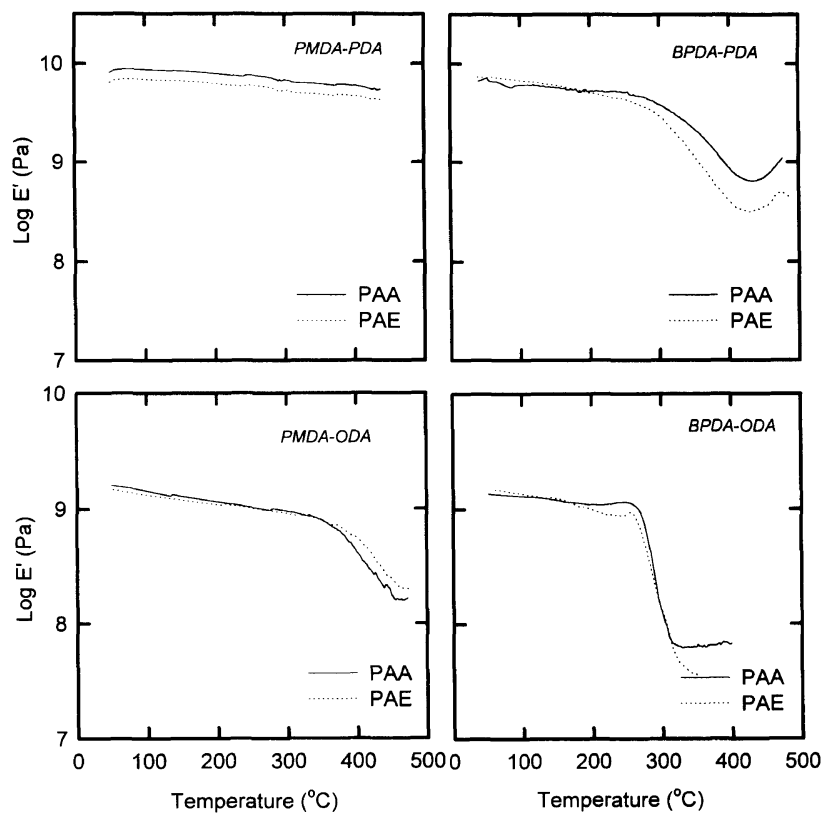

Figure 9. Variation of dynamic storage moduli in PAA-derived films and PAE-derived films with a heating rate of $5 \mathrm{Cmin}^{-1}$ at $1 \mathrm{~Hz}$.

morphological structure of BPDA-ODA. Regardless of precursor origin, BPDA-ODA revealed crystalline like diffraction with a big amorphous halo as shown in Figure $8(\mathrm{~b})$. From the diffraction patterns, it is expected that BPDA-ODA is a crystal structure like BPDA-PDA. The absence of the $(00 l)$ peak, which appeared in the transmission for others polyimides, is evidence that the polymer chains are randomly aligned in the film plane. For BPDA-ODA, transmission and reflection apparently were not influenced by precursor origin. This indicates that for the film from the PAE, the chain order and orientation in the BPDA-ODA polyimide chains was not different with the film from the PAA. From these results, it means that the morphological structure term, which includes molecular order and chain orientation, may play an important role in residual stress.

Information about the morphological structures was obtained from WAXD of the polyimides as shown in Figures 5 and 8. For the rigid PMDA-PDA and semirigid BPDA-PDA, the effect of different size of evaporated molecule in precursors through thermal imidization was significant to the degree of molecular orientation and chain ordered phase in fully cured polyimide. However, for semi-flexible PMDA-ODA and flexible BPDA-ODA, the effect of precursor origin on morphological structure was negligible.

Regardless of precursor origin, the residual stress of the fully cured polyimide thin films is in the increasing order $\mathrm{BPDA}-\mathrm{ODA}>\mathrm{PMDA}-\mathrm{ODA}>\mathrm{BPDA}-\mathrm{PDA}>$ PMDA-PDA. Coherence length, as critical factor to chain rigidity, and intermolecular chain order are in increasing order PMDA-PDA > BPDA-PDA > PMDA-ODA. No $(00 l)$ peak appeared in highly flexible BPDA-ODA. The degree of chain rigidity may be the predominant factor for residual stress of polyimide thin film.

In addition to the morphological structure such as molecular order and orientation, chain mobility should be considered as a key factor influencing residual stress behavior according to curing temperature. Chain rigidity in polyimide is directly related to the chain mobility and $T_{\mathrm{g}}$ in polyimide. $T_{\mathrm{g}}$ 's of the polyimide were examined as shown in Figure 9. The ethyl ester groups slightly affect storage modulus profiles for the polyimides. From the storage modulus profiles, onset temperatures of glass transition $\left(T_{\mathrm{g}}\right)$ were estimated to be $340^{\circ} \mathrm{C}$ for BPDA-PDA PAA, $330^{\circ} \mathrm{C}$ for BPDA-PDA PAE, $380^{\circ} \mathrm{C}$ for PMDA-ODA, and $285^{\circ} \mathrm{C}$ for BPDA-ODA regardless of precursors. PMDA-PDA did not show any glass transition over $50-400^{\circ} \mathrm{C}$, indicating its $T_{\mathrm{g}}$ higher than $400^{\circ} \mathrm{C}$.

During thermal imidization, the imidized chain mobility of PMDA-PDA is highly limited because $T_{\mathrm{g}}$ is higher than the imidization temperature. The storage modulus of PMDA-PDA polyimide is always higher than that of other polyimides, indicating PMDA-PDA is the most rigid polyimide. High residual stress at $400^{\circ} \mathrm{C}$ in PMDA-PDA might be contributed by this limited chain mobility due to high $T_{\mathrm{g}}$ and chain rigidity, as shown in stress results. The storage modulus of BPDA-PDA polyimide is always higher than that of PMDA-ODA and BPDA-ODA, indicating that the chain of BPDAPDA is relatively less mobile. However, the storage modulus of BPDA-PDA polyimide is relatively lower than that of PMDA-PDA. Chain mobility of the polyimide thin films during thermal imidization increase in the order BPDA-ODA > PMDA-ODA > BPDAPDA $>$ PMDA-PDA. Good agreement was shown with the results of residual stress and chain orientation estimated by WAXD in polyimide thin films.

Depending upon precursor origin in rigid polyimide such as PMDA-PDA and BPDA-PDA, the storage modulus is higher in films prepared from PAA than that of PAE-derived film. The higher storage modulus is induced from lower chain mobility in polyimide. The degree of chain mobility in rigid polyimide may be slightly higher in polyimide prepared from the PAE than that from the PAA. Limited chain mobility in PMDAPDA and BPDA-PDA prepared from PAA precursor may restrict sufficient stress development on cooling. Thus, more or less low storage modulus in PMDAPDA and BPDA-PDA prepared from PAE leads to higher residual stress in fully cured polyimide thin films as shown in Figure 9. However, regardless of precursor origin the variation of the storage modulus in PMDA-ODA and BPDA-ODA polyimides was not significant. These flexible PMDA-ODA and BPDAODA polyimides have relatively similar chain mobility regardless of precursor origin. Thus, for the PMDAODA and BPDA-ODA polyimides regardless of precursor origin, similar chain mobility might contribute to the same degree of residual stress after fully cured at $400^{\circ} \mathrm{C}$ as shown in Figures 6 and 7. Chain mobility of PMDA-PDA and BPDA-PDA polyimides, which have relatively high chain rigidity, is influenced significantly by the history of their precursor origin. However, the chain mobility of both PMDA-ODA and BPDA-ODA polyimides with low chain rigidity is little varied with the precursor origin. This shows good agreement with results of residual stress behavior and morphological structure of polyimide film. 


\section{CONCLUSION}

Fully rodlike PMDA-PDA, pseudo-rodlike BPDAPDA, and semiflexible PMDA-ODA and BPDA-ODA were investigated. Polyimides prepared from PAA and PAE were used. Residual stress of polyimide thin films was in situ monitored using a wafer bending method during thermal imidization and subsequent cooling at $25-400^{\circ} \mathrm{C}$. For polyimides prepared from the PAE precursors, residual stress of fully cured films at $400^{\circ} \mathrm{C}$ was 13.4 MPa for PMDA-PDA, 19.5 MPa for BPDAPDA, $30.3 \mathrm{MPa}$ for PMDA-ODA, and $39.5 \mathrm{MPa}$ for BPDA-ODA. For polyimides prepared from the PAA precursors, residual stress at $25^{\circ} \mathrm{C}$ after fully cured at $400^{\circ} \mathrm{C}$ was $-5.1 \mathrm{MPa}$ for the PMDA-PDA, 7.0 MPa for BPDA-PDA, 29.4 MPa for PMDA-ODA, and 40.1 MPa for BPDA-ODA. That is, the order of increasing chain rigidity of polyimide, which is estimated by coherence length, is PMDA-PDA > BPDA-PDA > PMDA-ODA $>$ BPDA-ODA, regardless of precursor origin. Residual stress of a polyimide film primarily depends on the chain rigidity in polymer. The effect of bulky size of precursor (PAE) on the residual stress behavior is significantly high in the rodlike PMDA-PDA or pseudo-rodlike BPDA-PDA polyimides having high chain rigidity and a high intermolecular packing order, but relatively low in the semiflexible PMDA-ODA and BPDA-ODA polyimides with relatively low chain rigidity. Residual stress in the polyimides was significantly related to morphological structure as well as chain mobility during thermal imidization of the polyimide as shown in WAXD and DMTA analysis. Degraded molecular orders by outgassing bulky size in polyimide film accelerated thermal mismatch with substrate and induced high the residual stress of fully cured PMDA-PDA and BPDA-PDA prepared from bulky size of precursor (PAE).

Acknowledgment. This work was supported in part by grant No. KOSEF 96-0200-06-01-3, and by grant No.
KOSEF 971-1102-007-2 from Korea Science and Engineering Foundation.

\section{REFERENCES}

1. K. L. Mittal, "Polyimides: Synthesis, Characterization and Application," Plenum Press, New York, N.Y., 1984.

2. D. Wilson, H. D. Stenzenberger, and P. M. Hergenrother. "Polyimides", Chapman \& Hall, New York, N.Y., 1990.

3. M. I. Bessonove, M. M. Koton, V. V. Kudryyavtsev, and L. A. Laius, "Polyimides: Thermally Stable Polymer," Consultants Bureau, New York, N.Y., 1987.

4. F. M. Houlihan, B. J. Bachman, Jr., C. W. Wilkins, and C. A. Pryde, Macromolecules, 22, 4477 (1988).

5. M. Udea and H. Mori, Macromol. Chem., 194, 511 (1993).

6. S. I. Kim, S. M. Pyo, and M. Ree, Macromolcules, 30, 7890(1997).

7. C. A. Pryde, J. Polym. Sci., Part A, Polym. Chem., 27, 711 (1989).

8. J. Brekner and C. Feger, J. Polym. Sci., Part A, Polym. Chem., 25, 2005 (1987).

9. G. Elsner, J. Appl. Polym. Sci., 34, 815 (1987).

10. J. H. Jou. P. T. Huang, H. C. Chen, and C. N. Liao, Polymer, 33, 967 (1990).

11. J. H. Jou. C. P. Lin, and W. H. Sheu, J. Polvm. Sci., Part B, Polvm. Phys., 33, 1803 (1995)

12. H. Nomura, M. Eguchi, and M. Asano, J. Appl. Phys., 70, 7085 (1991).

13. M. Ree, T. L. Nunes, G. Czornyj, and W. Volksen, Polymer, 33, 1228 (1992)

14. M. Ree, C. W. Chu, and M. J. Goldberg, J. Appl. Phys., 75, 1410 (1994).

15. Y. T. Chern and B. S. Wu, J. Appl. Polym. Sci., 61, 1853 (1996).

16. H. Han and M. Ree, Kor. Polym. J., 5, 152 (1997).

17. S. P. Timoshenko, J. Opt. Soc. of Am., 11, 223 (1926).

18. J. J. Wortman and R. A. Evans, J. Appl. Phys., 36, 136 (1965).

19. B. D. Cullity, "Elements of X-Ray Diffraction," 2nd ed, Addison-Wesley Publ. Co., Inc., .London, 1978.

20. J. H. Joe and P. T. Huang, Macromolecules, 24, 3796 (1991).

21. N. Takahashi, D. Y. Yoon, and W. Parrish, Macromolecules, 17, 2583 (1994).

22. M. Ree, S. Swanson, and W. Volksen, Polymer, 34, 1423 (1993).

23. M. Ree, T. J. Shin, Y. H. Park, S. I. Kim, S. H. Woo, C. K. Cho, and C. E. Park, J. Polym. Sci., Polym. Phys., 36, 1261 (1998).

24. H. Han, H. Chung, C. C. Gryte, T. J. Shin, and M. Ree, Polymer, 40, 2681 (1999).

25. H. Han, M. Ree, S. M. Pyo, and C. C. Gryte, Polymer, 39, 2963 (1998). 